\title{
Nanofabrication of Multi-Layer Devices and Multiple Writing Fields Using Focused Ion Beam and DualBeamTM Instruments
}

\author{
O. Wilhelmi, A. Rosenthal, P. Faber, F. Morrissey and L. Roussel
}

FEI Company, Achtseweg Noord, PO Box 80066, 5600 KA Eindhoven, The Netherlands

The capabilities of focused ion beams (FIB) to remove and deposit materials accurately on a nanometer scale are employed for prototyping purposes across all application areas of nanotechnology. The great advantage of doing nanoprototyping in a DualBeam instrument is that one single instrument is capable of directly writing a given pattern design in any kind of substrate material with the opportunity to monitor the patterning process live with an SEM. Being able to see a nanostructure evolving and inspect the result in great detail at the moment the patterning process is completed, allows rapid process optimization and delivers in many cases a first functional prototype of a nanodevice within a very short time.

The success of FIB nanoprototyping of single devices consisting of a small number of individual pattern elements [1]-[3] has led to a demand to expand the range of applications to large and complex patterns. Figure lexemplifies the integration of electric functionality into a nanofluidic device. The nanofluidic channel was milled into a glass substrate first and then an interdigitated Ptelectrode was deposited into the meanders of the nanofluidic channel. The proximity of electrical and fluidic structure requires precise pattern placement of both device layers. In order to enable process automation with the potential to replicate the device at different sites on the same substrate, an automated pattern alignment is executed which recognizes the four bright reference positions in figure land positions each pattern layer relative to them, correcting any shifts that might occur during insertion and retraction of the Pt gas injection system or the change of FIB currents.

Figure 2 shows how the technique of automated pattern alignments can be used for stitching of photonic crystal patterns. Photonic crystals that can not be patterned in a single writing field require stage moves to cover the entire pattern area. Mechanical tolerances during the stage moves often break the periodicity of the photonic crystal with the consequence of transmission losses or device failure. The direct patterning with a FIB bears the unique opportunity to write alignment marks as part of the pattern in each individual field and read back these alignment marks after the stage move to the next field. This technique allows passing on the exact pattern position of the previous writing field to the actual writing field. Figure 2 shows a photonic crystal pattern consisting of three individual writing fields. The cross-shaped alignment marks that were used for stitching purposes also indicate where the photonic crystal was stitched together. The stitching error at the lower stitching position cannot be seen at the scale of the micrograph, whereas the upper stitching position shows the stitching error of $46 \mathrm{~nm}$ as a fine horizontal line.

References:

[1] J. Schrauwen, J. Van Lysebettens, T. Claes, K. De Vos, P. Bienstman, D. Van Thourhout, R. Baets, IEEE Photonics Technology Letters Vol. 30 (23), 2008

[2] L.D. Menard, S.A. Soper, K.L. Braun, C. Huang, J.M. Ramsey, 12th Intern. Conf. on Miniaturized Systems for Chemistry and Life Sciences, Oct 12-16, 2008, San Diego, CA

[3] A. Fernandez-Pacheco, J.M. De Teresa, R. Cordoba, M.R. Ibarra, Nanotechnology 19, 2008 


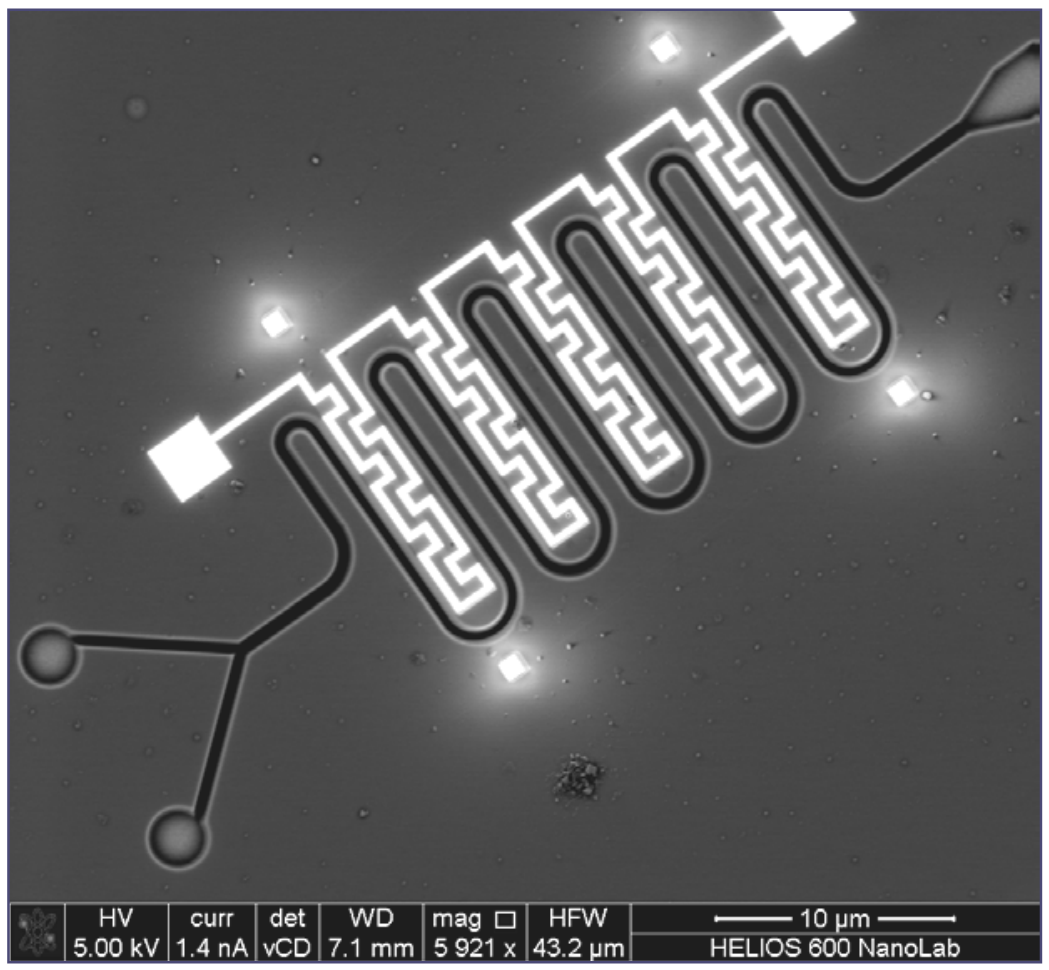

FIG. 1: A material contrast image of a nanofluidic channel (dark) in glass interdigitated with a Pt-electrode (bright).

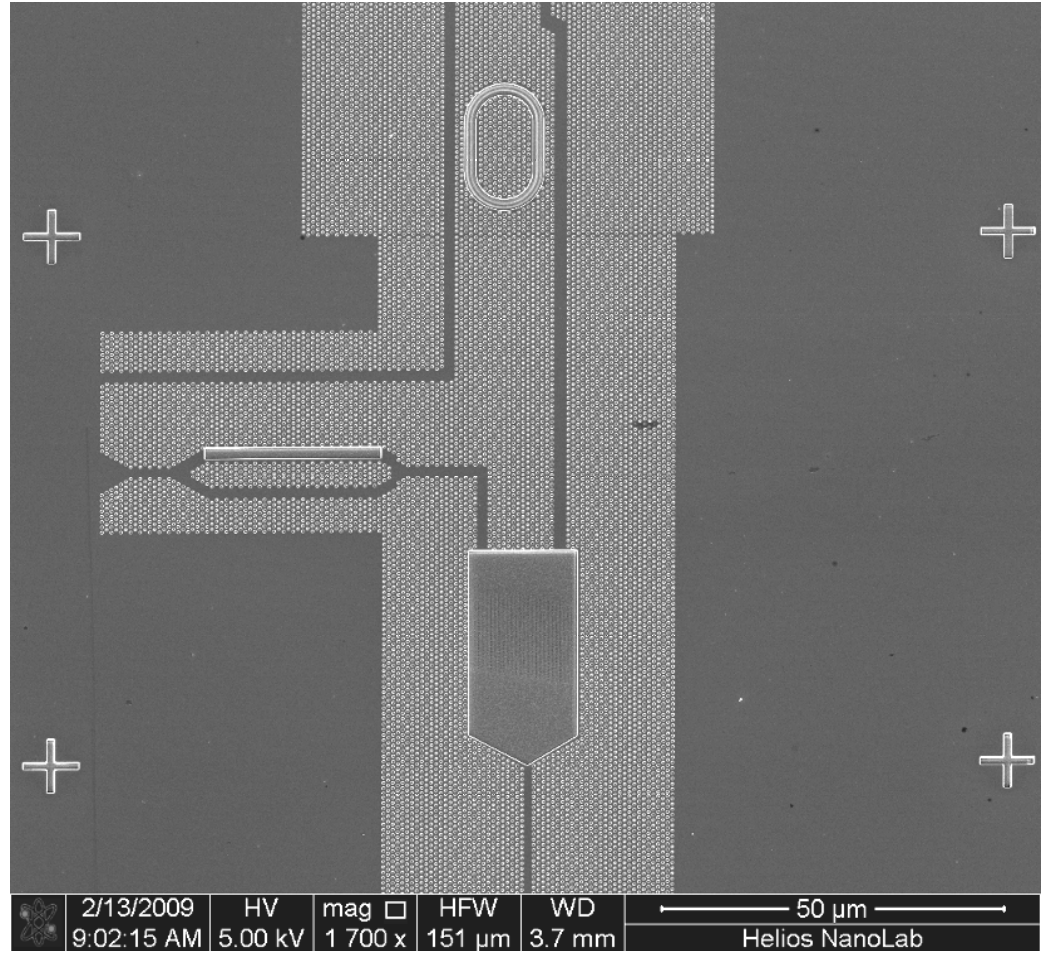

FIG. 2: Three writing fields of a photonic crystal that were patterned using automated alignment based on recognition of the cross-shaped alignment marks. 